\title{
The Nyquist Issue in Linear Inverse Modeling
}

\author{
CÉCILE PENLAND \\ NOAA/ESRL/Physical Sciences Division, Boulder, Colorado
}

(Manuscript received 26 March 2018, in final form 5 February 2019)

\begin{abstract}
Linear inverse modeling (LIM) is a statistical technique based on covariance statistics that estimates the best-fit linear Markov process to a multivariate time series. An integral, often-ignored part of the technique is a test of whether or not the linear assumptions are valid. One test for linearity is the so-called tau test. While this test can be trusted when it passes, it sometimes fails when it ought to pass. In this article, we discuss one of the reasons for spurious failure, the "Nyquist issue," which occurs when the lagged covariance matrix used in the analysis is numerically performed at a lag greater than or nearly equal to half the period of a natural mode of variability represented in the time series. As an illustration relevant to a system with many degrees of freedom, but simple enough to solve analytically, we consider a four-dimensional system consisting of two modal pairs. Within this framework, we suggest one solution that can be applied if the time series are long enough. It is hoped that awareness of this issue can prevent misinterpretation of LIM results.
\end{abstract}

\section{Introduction}

Linear inverse modeling (LIM) is an empirical technique of estimating the best linear stochastic model consistent with a set of multivariate data (Penland 1989; Penland and Sardeshmukh 1995a). As with autoregressive processes, LIM combines the lagged and contemporaneous covariance matrices to estimate the relevant product matrices. However, unlike autoregressive processes, LIM estimates the operator in a continuous stochastic differential equation as well as the correlation structure of the driving white noise. These operators can then be used to diagnose whether or not the governing dynamical system may be described as a linear stochastic differential equation with constant coefficients. Once linearity has been established, forecasts with a theoretically estimated error can be made, and a variety of analyses can be performed to investigate the dynamical relationships between the constituent variables. Of course, these analyses depend on the fidelity of a linear model to the real dynamical system; the mechanics of the analysis, after all, can be applied to any multivariate time series, regardless of the underlying dynamics.

Assessing this fidelity requires a strict test for the validity of LIM, and LIM is valid for time series generated by dynamical systems on a time scale where nonlinear

Corresponding author: Cécile Penland, cecile.penland@noaa.gov interactions are so fast and so chaotic that they may be approximated by Gaussian white noise terms in a limiting differential equation. That is, the ultimate source of what a coarse-grained system sees as Gaussian white noise may be neither Gaussian nor white at some (unresolved) time scale. The integrated effect of rapidly varying nonlinearities is subject to the central limit theorem, so that a more slowly varying component of the multiscale system cannot be distinguished from a process driven by Gaussian white noise when represented by a coarse-grained time series (Papanicolaou and Kohler 1974; Hasselmann 1976).

As time series analysis is inherently statistical, testing for linearity is also statistical. Ideally, this test would pass only when the generating dynamical system is linear and fail only when it is not. In practice, however, statistical tests have a (hopefully small) probability of delivering a false result. Here we consider a test for linearity, the "tau test" (e.g., Penland 1989), against a null hypothesis that the dynamical system generating a multivariate time series is nonlinear on the time scales of interest, that is, that nonlinearities cannot be estimated as a linear process with either additive or multiplicative Gaussian white noise. The tau test consists of estimating linear operators based on the lagged covariance matrices at a variety of lags $\tau_{o}$ (e.g., Penland and Sardeshmukh 1995a; Winkler et al. 2001; Shin et al. 2010; Liu et al. 2012a,b). Linear operators so estimated are expected to be independent of $\tau_{o}$. This tau test is generally trustworthy when it 
passes; Penland and Sardeshmukh (1995a), for example, have shown the spectacular failures of this test when applied to the Lorenz (1963) system in its chaotic regime. Unfortunately, the tau test is notorious for also failing in some cases when the underlying dynamics should indeed be approximated as linear.

It has been shown that the tau test can spuriously fail for several reasons. If the statistics are nonstationary, the tau test will fail (Penland and Sardeshmukh 1995a). If the state vector under consideration does not represent all of the important variables, the tau test will fail (Penland and Ghil 1993). If the time series is seriously corrupted with observation errors, the tau test will fail (Penland 1998). Perhaps the most frustrating cause of tau-test failure, however, is the near inevitability of "Nyquist lags" (Penland and Sardeshmukh 1995a, their appendix B). A Nyquist lag occurs when the lag $\tau_{o}$ of the covariance matrix used to estimate the linear operator is near half the period of an intrinsic oscillatory mode of the system. Then, that mode cannot be accurately resolved numerically. If this mode is an important constituent of the dynamics, the numerically estimated linear operator is significantly corrupted, and the tau test fails.

When LIM is applied to geophysically relevant datasets, the large number of degrees of freedom requires numerical eigenanalysis, and the Nyquist problem is inevitable. It is the purpose of this article to discuss the Nyquist problem in LIM and to show how it can be resolved in a system that is simple enough to be solved analytically, but where the presence of multiple oscillatory modes requires a sequential, mode-by-mode method of handling the problem. We do not claim to solve the problem in such a general way that it is easy to automate. However, as shown below, this solution can be useful for real world problems, such with a reduced model of El Niño. We first review LIM and present the difficulties associated with the Nyquist problem. This section includes a possible solution to the problem described step by step for a single modal pair. The next section, example 1 , shows how the mode-by-mode procedure can be applied to a simple four-dimensional system (two modal pairs) where the analytical solution is known. This section describes in detail both how the estimated linear operator can be corrupted when the unmodified modes are combined and how the linear operator is recovered by proper adjustment of the modes. We also show the kind of evidence that would suggest there is a Nyquist problem in the first place. Finally, to illustrate practical problems, we apply the Nyquist correction to a reduced, empirically derived model of El Niño. The formalism and terminology throughout this article are similar to those used in many previous publications by the American Meteorological Society (e.g., Farrell and Ioannou 1993; Penland and Sardeshmukh 1995a,b; DelSole 1996; Sheshadri et al. 2018, and references therein). Relevant theoretical details concerning matrix methods, for example, the Cayley-Hamilton theorem referenced below, may be found in the textbook by Bronson (1970).

\section{Review of LIM and the Nyquist problem}

Consider an $M$-dimensional vector $\mathbf{x}(t)$, the $i$ th component $x_{i}(t)$ of which represents a zero-mean observation at location $i$ and time $t$. We wish to show whether the governing dynamics of this system obeys a linear Markov process. In this study, we consider only additive noise, that is, a stable linear process called a multivariate Ornstein-Uhlenbeck (OU) process. The equation for an OU process is

$$
\frac{d \mathbf{x}}{d t}=\mathbf{L x}+\boldsymbol{\xi},
$$

where $\mathbf{L}$ is a constant matrix and where $\boldsymbol{\xi}$ is a white noise vector with covariance matrix $\left\langle\boldsymbol{\xi} \boldsymbol{\xi}^{\mathrm{T}}\right\rangle d t=\mathbf{Q}$, and where angle brackets indicate ensemble average. Note that Eq. (1) integrated over a unit time step is equivalent to a first-order autoregressive model $\mathbf{x}(t)=\mathbf{A} x(t-1)+\boldsymbol{\varepsilon}$, but with specific functional forms for $\boldsymbol{\varepsilon}$ and $\mathbf{A}$. If $\mathbf{Q}$ is constant, the statistics of $\mathbf{x}(t)$ are stationary and we may treat the time average as the ensemble average. If $\mathbf{Q}$ is periodic with period $T$, then the statistics of $\mathbf{x}(t)$ are also periodic with period $T$ and care must be taken in the estimation of ensemble averages. For $\mathbf{Q}$ stationary, the lagged autocovariance matrix of $\mathbf{x}$ is related to the contemporaneous autocovariance matrix as

$$
\left\langle\mathbf{x}\left(t+\tau_{o}\right) \mathbf{x}^{\mathrm{T}}(t)\right\rangle=\exp \left(\mathbf{L} \tau_{o}\right)\left\langle\mathbf{x}(t) \mathbf{x}^{\mathrm{T}}(t)\right\rangle .
$$

For $\mathbf{Q}$ periodic, Eq. (2) holds with the angle brackets now including a time average over an integer number of periods $T$. If $\mathbf{L}$ itself is a function of time, of course, the tau test will fail. For expository purposes, we will hereafter assume stationary statistics unless otherwise indicated. We also use the notation $\mathbf{C}(\tau) \equiv\left\langle\mathbf{x}(t+\tau) \mathbf{x}^{\mathrm{T}}(t)\right\rangle$.

As suggested by Eq. (2), we use the time series to estimate the Green function matrix $\mathbf{G}\left(\tau_{o}\right)$ :

$$
\mathbf{G}\left(\tau_{o}\right)=\mathbf{C}\left(\tau_{o}\right) \mathbf{C}(0)^{-1} .
$$

Note that when $\tau_{o}=1$, Eq. (3) is also starting point of principal oscillation pattern analysis (Von Storch et al. 1988). Since $\mathbf{G}\left(\tau_{o}\right)$ and $\mathbf{L}$ have the same eigenmodes $\left\{\mathbf{u}_{\alpha}\right\}$ and adjoints $\left\{\mathbf{v}_{\alpha}\right\}, \mathbf{G}\left(\tau_{o}\right)$ and $\mathbf{L}$ can be similarly expanded as a sum over the modal index $\alpha$ from 1 to $N$, where $N$ is the number of modes: 


$$
\begin{aligned}
\mathbf{G}\left(\tau_{o}\right) & =\sum_{\alpha=1}^{N} \mathbf{u}_{\alpha} g_{\alpha}\left(\tau_{o}\right) \mathbf{v}_{\alpha}^{\mathrm{T}}, \quad \text { and } \\
\mathbf{L} & =\sum_{\alpha=1}^{N} \mathbf{u}_{\alpha} \beta_{\alpha} \mathbf{v}_{\alpha}^{\mathrm{T}},
\end{aligned}
$$

where $g_{\alpha}\left(\tau_{o}\right)$ is the eigenvalue of $\mathbf{G}\left(\tau_{o}\right)$ corresponding to eigenmode $\mathbf{u}_{\alpha}$ and of $\mathbf{G}^{\mathrm{T}}\left(\tau_{o}\right)$ corresponding to adjoint $\mathbf{v}_{\alpha}$, and where $\beta_{\alpha}$ is the eigenvalue of $\mathbf{L}$ corresponding to eigenmode $\mathbf{u}_{\alpha}$ and of $\mathbf{L}^{\mathrm{T}}$ corresponding to adjoint $\mathbf{v}_{\alpha}$. Because the matrices $\mathbf{G}\left(\tau_{o}\right)$ and $\mathbf{L}$ satisfy their respective characteristic equations (Hamilton 1853; Cayley 1858; Bronson 1970), their eigenvalues are related in the same way that they are, that is,

$$
g_{\alpha}\left(\tau_{o}\right)=\exp \left(\beta_{\alpha} \tau_{o}\right)
$$

Thus, the eigenvalue $\beta_{\alpha}$ can be estimated by taking the complex natural logarithm of $g_{\alpha}\left(\tau_{o}\right)$ and dividing by $\tau_{o}$, after which $\mathbf{L}$ is constructed using Eq. (4b). Note that Eqs. (4) are valid if the eigenmodes and adjoints are normalized so that they form a binormal set, that is, if $\mathbf{u}_{\alpha}^{\mathrm{T}} \mathbf{v}_{\beta}=\delta_{\alpha \beta}$.

One version of the tau test consists of checking that the eigenvalues $\beta_{\alpha}$ are independent of $\tau_{o}$ (see below). Another version considers the norm of $\mathbf{L}$ itself (e.g., Shin et al. 2010; Liu et al. 2012a,b). Yet another version uses the expansion Eq. (4a) to generate the Green function $\mathbf{G}(\tau)$ at other lags using the relation

$$
g_{\alpha}(\tau)=\left[g_{\alpha}\left(\tau_{o}\right)\right]^{\tau / \tau o}
$$

and then compares the expected autocovariance map at that lag with the autocovariance estimated directly from the time series (e.g., Winkler et al. 2001). A related version involves comparison of the power spectrum estimated from the measured time series with that estimated from ensemble members generated numerically from the results of LIM applied to that time series (Newman 2007). One can also compare forecast errors estimated with sets of Green functions estimated at different lags (e.g., Penland and Sardeshmukh 1995a).

It is here that the Nyquist issue arises. The eigenvalues $\left\{g_{\alpha}\left(\tau_{o}\right)\right\}$ are either real, or they and their associated eigenvectors occur in complex conjugate pairs. Recall that $\mathbf{L}$ is a stable, real, linear operator, and its eigenvalues $\left\{\beta_{\alpha}\right\}$ are either real and negative, or complex with negative real part. That is, the deterministic evolution of the complex eigenmode consists of exponential decay with a rate $\operatorname{Re}\left(\beta_{\alpha}\right)$ and an oscillation with angular frequency $\operatorname{Im}\left(\beta_{\alpha}\right)$. These values are estimated using the complex logarithm of $g_{\alpha}\left(\tau_{o}\right)$, which is multivalued. Hence, for $\tau_{o}$ close to $\pi / \operatorname{Im}\left(\beta_{\alpha}\right)$, an automated eigenanalysis system will not be able to identify the imaginary part of the mode. We thus call $\pi / \operatorname{Im}\left(\beta_{\alpha}\right)$ a Nyquist lag, and there are as many Nyquist lags as there are complex modal pairs. For $\tau_{o}$ larger than $\pi / \operatorname{Im}\left(\beta_{\alpha}\right)$, the automated eigenanalysis package returns an estimated eigenvalue $g_{\alpha}^{\prime}\left(\tau_{o}\right)=\exp \left[\beta_{\alpha} \tau_{o} \pm(2 \pi n) i\right]$ or its complex conjugate, the logarithm of $g_{\alpha}^{\prime}\left(\tau_{o}\right)$ divided by $\tau_{o}$ is not constant, and the tau test fails. The quadrant of the complex plane in which the automated package locates $g_{\alpha}^{\prime}\left(\tau_{o}\right)$ may depend on the eigenanalysis package or perhaps even the compiler.

At first, the situation does not seem so grave. After all, if the eigenmode and adjoint do not change, it seems that all one would have to do is adjust the eigenvalue appropriately and proceed with the expansion Eq. (4b). However, things are a little more complicated. Once $\tau_{o}$ is big enough to change the $\operatorname{sign}$ of $\sin \left[\operatorname{Im}\left(\beta_{\alpha} \tau_{o}\right)\right]$, the multivalued nature of the inverse trigonometric functions comes into play. Say, for example, the decay time of the $\alpha$ th eigenvalue is 5 time units (tu) and its period is $10 \mathrm{tu}$. That is, say the eigenvalue $g_{\alpha}\left(\tau_{o}=4 \mathrm{tu}\right)$ is $\exp [(-0.2+2 \pi i / 10) 4]$ and $g_{\alpha+1}\left(\tau_{o}=4 \mathrm{tu}\right)$ is its complex conjugate. The automated eigenanalysis routine returns the complex value $g_{\alpha}\left(\tau_{o}\right)=\exp (-0.8)\{\cos (8 \pi i / 10)+i \sin (8 \pi i / 10)\}$ associated with the mode $\mathbf{u}_{\alpha}$ At the same time, the eigenvalue associated with $\mathbf{u}_{\alpha+1}$ is $\exp (-0.8)\{\cos (8 \pi i / 10)-i \sin (8 \pi i / 10)\}$. Now, letting $\tau_{o}=8 \mathrm{tu}$, the routine returns $g_{\alpha}\left(\tau_{o}\right)=$ $\exp (-0.16)\{\cos (16 \pi i / 10)+i \sin (16 \pi i / 10)\}$. However, this equals $\exp (-0.16)\{\cos (2 \pi-8 \pi i / 10)-i \sin (2 \pi-8 \pi i / 10)\}$, which is numerically equivalent to $\exp (-0.16)\{\cos (8 \pi i / 10)-$ $i \sin (8 \pi i / 10)\}$. This eigenvalue is proportional to $g_{\alpha+1}\left(\tau_{o}=4 \mathrm{tu}\right)$, so the eigenanalysis routine interprets the eigenvector associated with it as $\mathbf{u}_{\alpha+1}$, the complex conjugate of $\mathbf{u}_{\alpha}$. When this happens, that is, for $\pi / \operatorname{Im}\left(\beta_{\alpha}\right) \leq$ $\tau_{o}<2 \pi / \operatorname{Im}\left(\beta_{\alpha}\right)$, the estimated mode $\mathbf{u}_{\alpha}^{\prime}$ and its estimated adjoint $\mathbf{v}_{\alpha}^{\prime}$ must be replaced by their complex conjugates to satisfy the tau test. More generally, the true mode $\mathbf{u}_{\alpha}=\mathbf{u}_{\alpha}^{\prime}$ for $2 \pi n / \operatorname{Im}\left(\beta_{\alpha}\right) \leq \tau_{o}<(2 n+1) \pi / \operatorname{Im}\left(\beta_{\alpha}\right)$, where $n$ is an integer, $\mathbf{u}_{\alpha}=\left(\mathbf{u}_{\alpha}^{\prime}\right) *$ for $(2 n+1) \pi / \operatorname{Im}\left(\beta_{\alpha}\right) \leq \tau_{o}<(2 n+2) \pi /$ $\operatorname{Im}\left(\beta_{\alpha}\right)$, and similarly with the adjoint.

\section{Example 1: Two noninteracting oscillators}

Our first example of applying the Nyquist correction concerns an extremely simple situation of a very long time series generated by two perfect noninteracting oscillators. The length of the time series and the simplicity of the linear operator were chosen in order to isolate the Nyquist problem from, for example, sampling issues. We numerically generate Eq. (1), specifying 


$$
\mathbf{L}=\left(\begin{array}{cccc}
-0.1 & 2 \pi / 14 & 0 & 0 \\
2 \pi / 14 & -0.1 & 0 & 0 \\
0 & 0 & -0.2 & -2 \pi / 10 \\
0 & 0 & 2 \pi / 10 & -0.2
\end{array}\right)
$$

and stochastic forcing such as to yield a time series with autocovariance matrix

$$
\mathbf{C}(0)=\left(\begin{array}{cccc}
8.75 & 1.25 & 0 & 0 \\
1.25 & 8.75 & 0 & 0 \\
0 & 0 & 5.25 & 0.75 \\
0 & 0 & 0.75 & 5.25
\end{array}\right)
$$

This is done using the stationary fluctuation-dissipation relation to estimate $\mathbf{Q}$ :

$$
-\mathbf{Q}=\mathbf{L C}(0)+\mathbf{C}(0) \mathbf{L}^{\mathrm{T}} .
$$

The multivariate time series was generated using a stochastic Euler scheme (Rümelin 1982) with a time step of (1/120) time units and output was sampled every time unit, that is, every 120 time steps, for a total of 50000 samples. To test for any sampling issues, calculations were performed with a time series half this long and yielded similar results. Sampling issues have been discussed elsewhere (Penland and Sardeshmukh 1995b; Penland and Matrosova 2001).

This operator $\mathbf{L}$ has four eigenvalues, $\beta_{1,2}=-0.1 \pm$ $i(2 \pi / 14)$ and $\beta_{3,4}=-0.2 \pm i(2 \pi / 10)$, associated with four eigenmodes that are the complex conjugates of their corresponding adjoints. Henceforth, we use $-\sigma_{\alpha}$ to denote $\operatorname{Re}(\beta \alpha)$ and $\omega_{\alpha}$ to denote $\operatorname{Im}(\beta \alpha)$. Note that we expect Nyquist lags at $\tau_{o}=5$ and 7 , and again at $\tau_{o}=10$ and 14 .

LIM was applied to this output using 15 values of $\tau_{o}$, that is, $\tau_{o}=1,2,3, \ldots, 13,14,15$. Figure 1 shows the Euclidean (L2) norm of the matrix $\mathbf{L}$ resulting from the raw estimation of its eigenstructure as a function of $\tau_{o}$ using Eqs. (3)-(5). The norm is constant with respect to $\tau_{o}$ and equal to the norm of the specified $\mathbf{L}$ until $\tau_{o}$ equals the smallest Nyquist lag, after which there is clear deviation from the specified value. Figure 2 a shows the raw estimation (blue dots) of $\sigma_{1,2}$ and $\left|\omega_{1,2}\right|$ compared with the specified values of $\sigma_{1,2}=0.1$ and $\left|\omega_{1,2}\right|=2 \pi / 14$. While $\sigma_{1,2}$ is well estimated at all lags $\tau_{o}$, the raw estimation of $\left|\omega_{1,2}\right|$ is accurate only up to the Nyquist lag $\tau_{o}=7$. Between $\tau_{o}=7$ and $\tau_{o}=14$, the correct value of $\left|\omega_{1,2}\right|$ is recovered by subtracting the estimated value $\omega_{e}$ from $2 \pi / \tau_{o}$, that is, $\left|\omega_{1,2}\right|=2 \pi / \tau_{o}-\omega_{e}$. Between $\tau_{o}=14$ and $\tau_{o}=18$, the correct value of $\left|\omega_{1,2}\right|$ is recovered by adding $2 \pi / \tau_{o}$ to the estimated value $\omega_{e}$, that is, $\left|\omega_{1,2}\right|=2 \pi / \tau_{o}+\omega_{e}$ (not shown here, but see Fig. 2b). Figure 2b is analogous to Fig. 2a, but for $\sigma_{3,4}$ and $\left|\omega_{3,4}\right|$, where the Nyquist lags shown here are at $\tau_{o}=5$ and 10 .

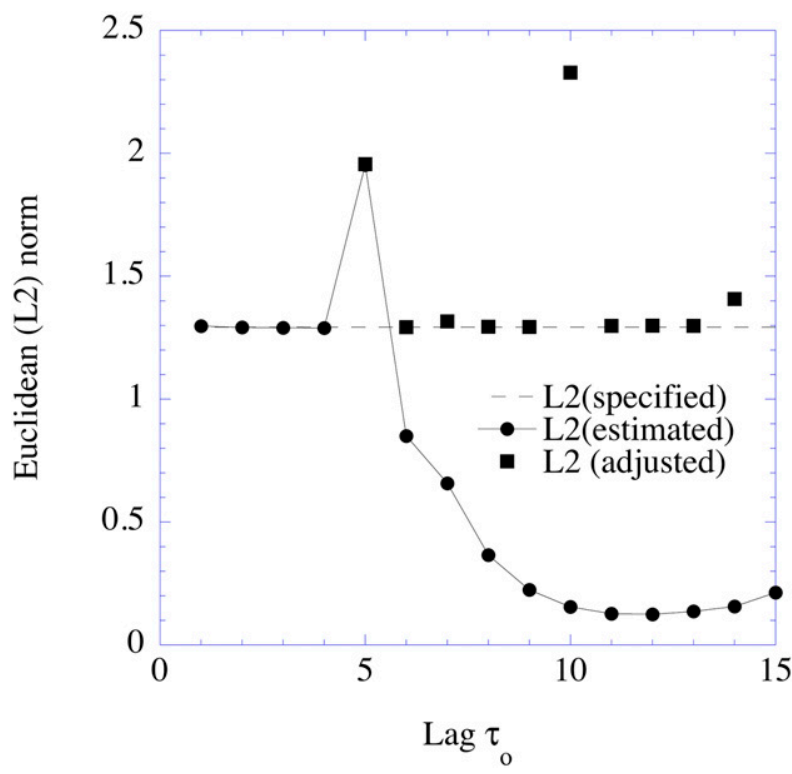

FIG. 1. Specified Euclidean norm of matrix $\mathbf{L}$ [Eq. (7a): dotted line]. Also shown: Euclidean norm of the raw estimation of $\mathbf{L}$ (filled circles) and of $\mathbf{L}$ after adjustment of modes and eigenvalues (filled squares).

Turning to the modes, we compare the estimated $\mathbf{u}_{1}$ as a function of $\tau_{o}$, for $\tau_{o}=3,8$, and 15 , with the analytically derived, normalized value of $\mathbf{u}_{1}=(1-i, 1+i, 0,0)^{\mathrm{T}} / 2$ in Fig. 3. As expected, the real part of $\mathbf{u}_{1}$ is well reproduced at all lags $\tau_{o}$, even though $\tau_{o}=15$ is larger than the decay time $\left(1 / \sigma_{1}\right)$ of that mode. The imaginary part of that eigenmode, however, undergoes a sign change at $\tau_{o}=8$ before reverting back to the derived value at $\tau_{o}=15$.

The same type of behavior is noted for $\mathbf{u}_{3}$ (Fig. 4), even though the decay time of this mode is less than half of $\tau_{o}=13$. The analytically derived expression for $\mathbf{u}_{3}$ is $\mathbf{u}_{3}=(0,0,1+i, 1-i)^{\mathrm{T}} / 2$. We note that the results for a value of $\tau_{o}$ so much larger than the decay time of the mode is only possible with very long time series. What generally happens with time series of realistic length is that at $\tau_{o}$ near the Nyquist lag, the imaginary part of the estimated eigenvalue $g_{\alpha}^{\prime}\left(\tau_{o}\right)$ is returned as $\pi$ and the imaginary part of the estimated mode $\mathbf{u}_{\alpha}$ is returned as zero.

Fortunately, the recombination of the operator $\mathbf{L}$ is independent of normalization. Figure 1 shows that the $\mathbf{L} 2$ norm of $\mathbf{L}$ for $\tau_{o}=1$ to 14 with the eigenvalues and eigenvectors properly adjusted agrees quite well with the specified value except at the Nyquist lags $\tau_{o}=5,7,10$, and 14. Of course, the L2 norm is independent of the sign of its elements, so we consider a stricter criterion. Figure 5 shows the bubble plots of $\mathbf{L}$ for the various cases. Here, the diameter of a circle rather than the area is proportional to the corresponding 

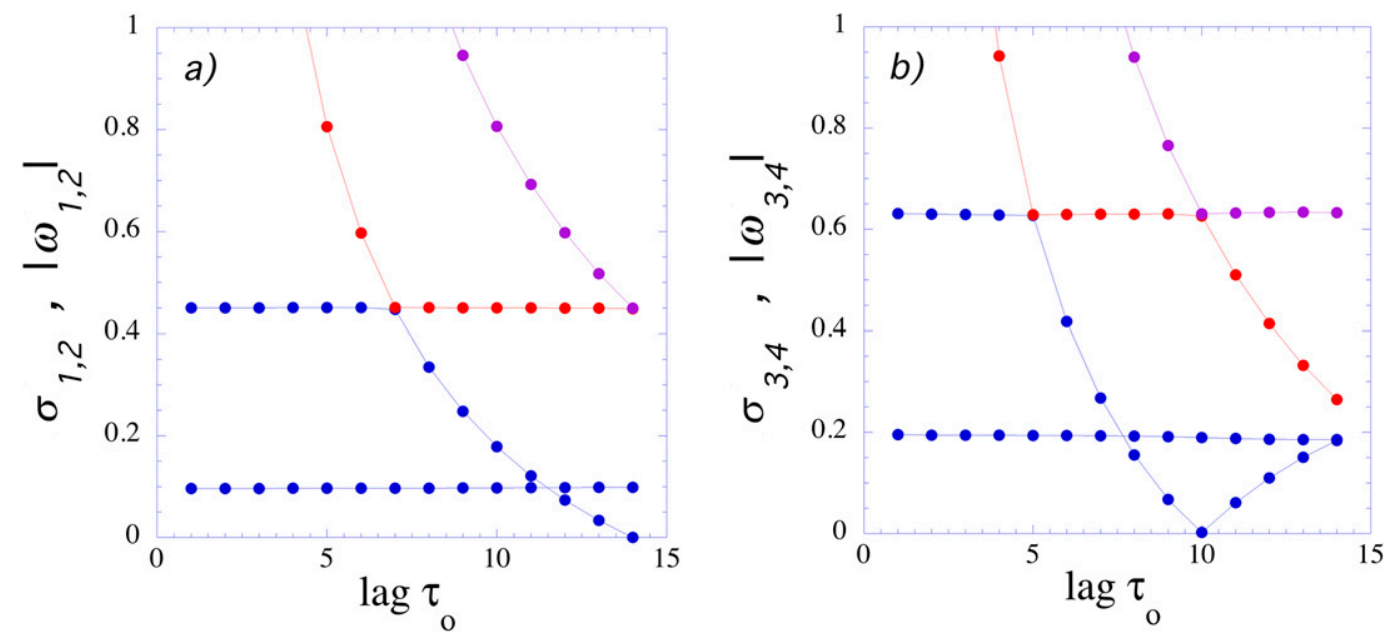

FIG. 2. Comparison of raw estimation of LIM eigenvalues (blue dots) with adjustment of imaginary parts, first by subtracting the estimated value from $2 \pi$ (red dots) and then by adding the estimated value to $2 \pi$ (purple dots).

(a) Decay rate and frequency of first modal pair. (b) Decay rate and frequency of second modal pair.

element of $\mathbf{L}$ in order to highlight the differences. Figures $5 \mathrm{a}-\mathrm{c}$ show the recombined $\mathbf{L}$ derived from the raw LIM results for $\tau_{o}=3,8$, and 13 . Figure $5 \mathrm{~d}$ shows the bubble plot of the specified L. Figs. 5 e, f show the recombined $\mathbf{L}$ derived from the results for $\tau_{o}=8,13$ after proper adjustments of the eigenvalues and eigenvectors.

Again, the results of this section were obtained with very long time series, and we note here the findings of Penland and Sardeshmukh (1995b) that recombined products of the eigenanalysis performed with LIM on time series of more usual length are more accurate than any of the individual modal products themselves.

\section{Example 2: A reduced model of EI Niño}

Penland and Matrosova (2006) showed that El Niño as represented in sea surface temperature (SST) data could be well approximated as a six-component system consisting of three empirical eigenmode pairs. This sixcomponent system, a subset of modes estimated using LIM applied to SSTs in the tropical belt, described the nonnormal evolution from an optimal initial condition to a mature El Niño pattern. It is not the purpose of this article to consider the El Niño phenomenon; rather, this six-component system is an ideal showcase for the uncertainties and Nyquist issues encountered when LIM is applied in a realistic situation.

LIM was applied to three-month running mean SST anomalies from the Comprehensive Ocean-Atmosphere Data Set (COADS) monthly SST data between 1950 and 1997 (Woodruff et al. 1993), concatenated with three years (1998-2000) of a real-time surface marine data product from the National Centers for Environmental Prediction (NCEP), for a total of 598 samples.
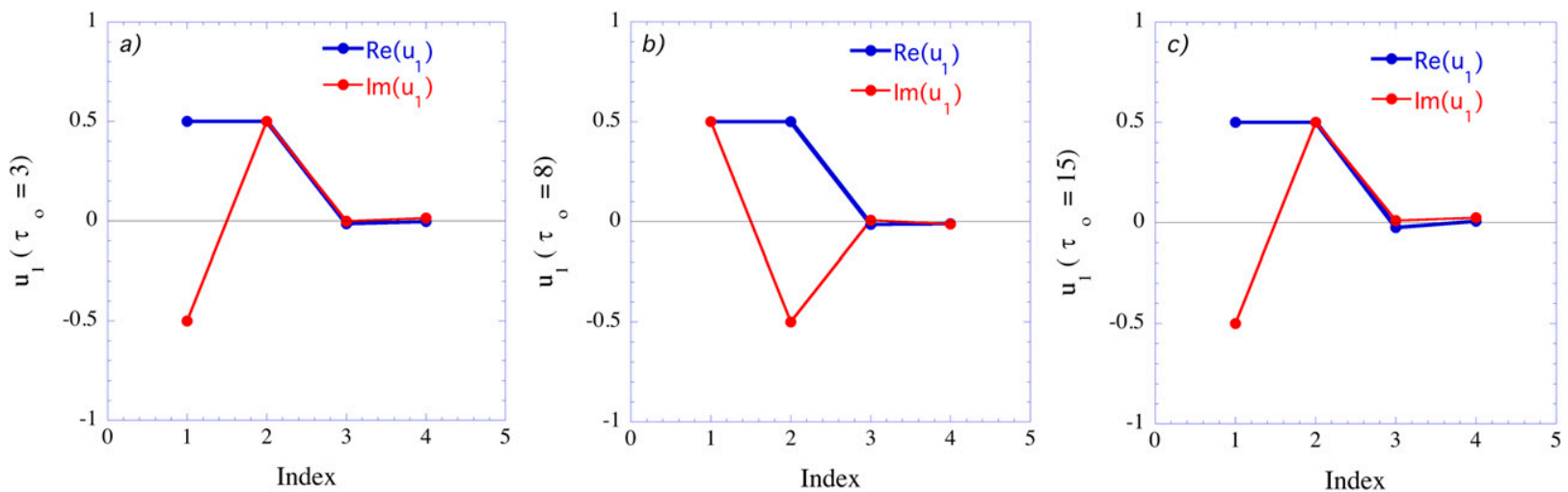

FIG. 3. Real (blue lines) and imaginary parts (red lines) of mode $\mathbf{u}_{1}$ estimated using (a) $\tau_{o}=3$, (b) $\tau_{o}=8$, and (c) $\tau_{o}=15$.

The analytically derived, normalized value of mode $\mathbf{u}_{1}$ is $(1-i, 1+i, 0,0)^{\mathrm{T}} / 2$. 

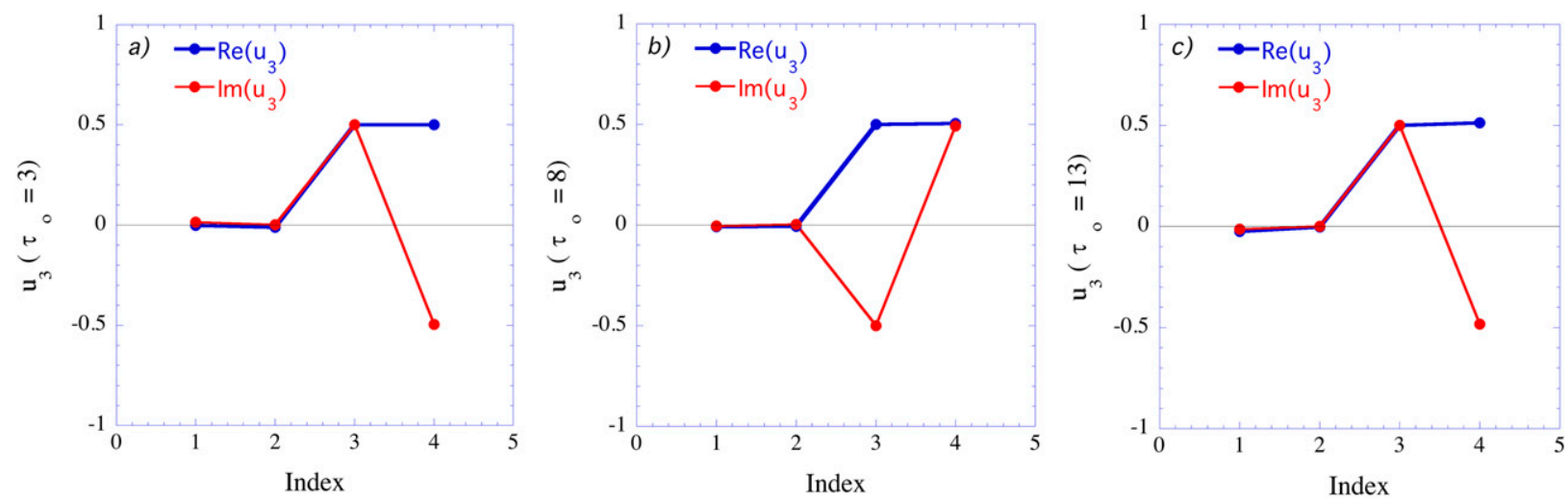

FIG. 4. As in Fig. 3, but for $\mathbf{u}_{3}$. The derived value of $\mathbf{u}_{3}$ is $(0,0,1+i, 1-i) / 2$.

The running mean was required to allow subsurface dynamical information time to migrate into the SST while maintaining a time series long enough to perform the LIM procedure. (Modern applications of LIM to El Niño take advantage of subsurface reanalyses which were not available to Penland and Sardeshmukh in 1995, on which the 2006 study was based.) Details of the data analysis are found in Penland and Matrosova (2006). The characteristics of this three-modal pair system as estimated using $\tau_{o}=4$ months are summarized in Table 1. Please note that the decay times associated with these modes are smaller than the periods. In particular, the decay time of the most rapidly oscillating mode (decay time $=6.8$ months; period $=23.3$ months) is more than three times smaller than the oscillation period. Nevertheless, we shall see that the time scales of this mode are reasonably independent of $\tau_{o}$ until lags are similar to the smallest Nyquist lag of about 11 months. LIM was applied to the data at values of $\tau_{o}=3-14$ months, and a plot of the L2 norm of the resulting linear operators shows significant violations of the tau test at about tau $=$ 10 months (Fig. 6), which we explain either as an artifact
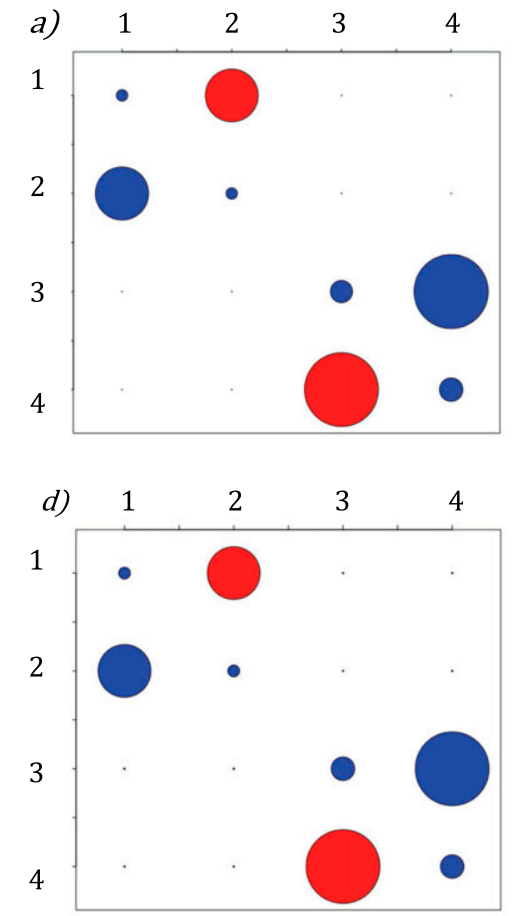
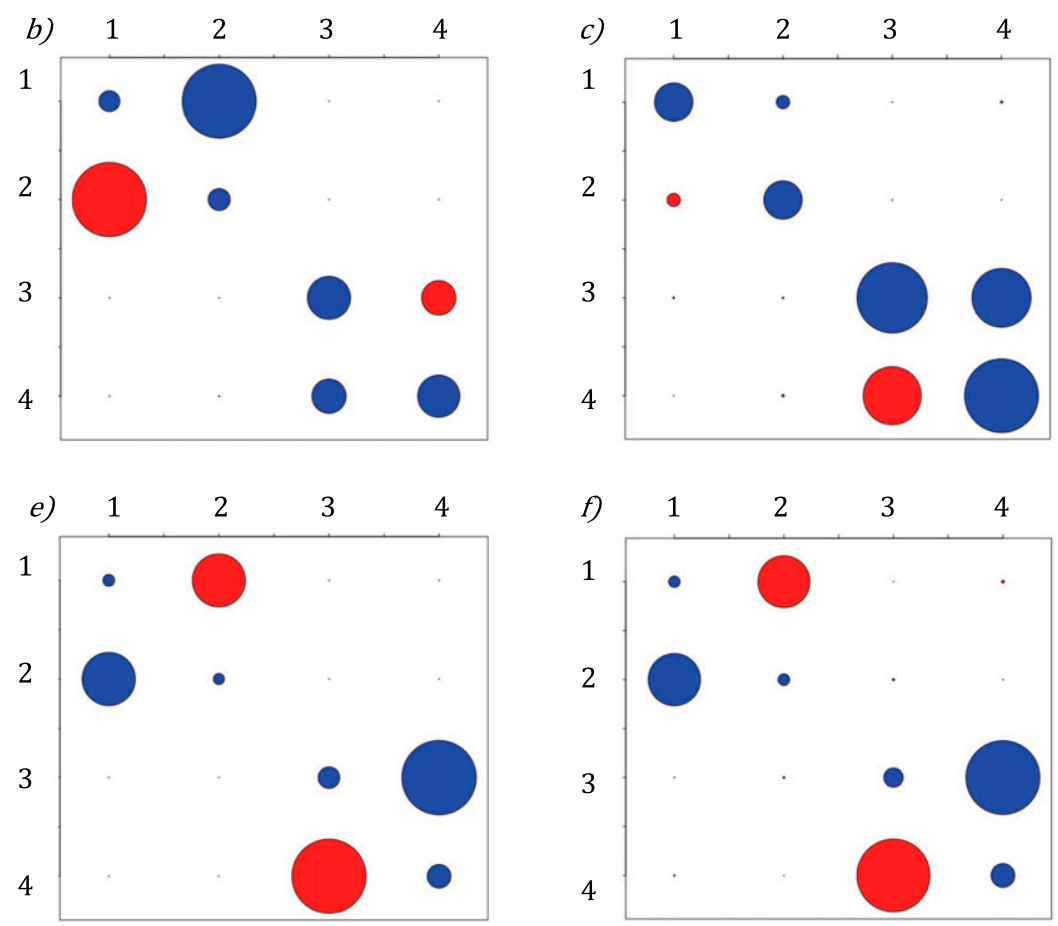

FIG. 5. Bubble plots representing specified [Eq. (7a)], estimated, and adjusted matrices L. Diameter is proportional to element size. Blue: negative elements. Red: Positive elements. (top) Estimated matrices using (a) $\tau_{o}=3$, (b) $\tau_{o}=8$, and (c) $\tau_{o}=13$. (bottom) (d) Specified matrix L. (e) Adjusted matrix for $\tau_{o}=8$. (f) Adjusted matrix for $\tau_{o}=13$. 
TABLE 1 . Time scales of empirically derived modes.

\begin{tabular}{ccc}
\hline \hline Modal pair & Decay time (months) & Period (months) \\
\hline $1 / 2$ & 15.6 & 223.3 \\
$3 / 4$ & 12.7 & 56.2 \\
$5 / 6$ & 6.8 & 23.3 \\
\hline
\end{tabular}

of the three-month running mean, to the inability of LIM to identify unambiguously the modes from a short time series, or both.

To test the vagaries of LIM applied to a time series of typical length, we use the constant matrices $\mathbf{L}$ and $\mathbf{Q}$ as estimated from the SST data using LIM at $\tau_{o}=4$ months to run an ensemble of 50 simulations of Eq. (1), each having 600 samples. Figure 7 reproduces the information in Fig. 6 along with the spread of L2 norms resulting from applying LIM to this ensemble at $\tau_{o}=4$ months and $\tau_{o}=10$ months. It is clear that uncertainties in the estimation of $\mathbf{L}$ become very large in a short time series as the Nyquist lag is approached, and that the L2 norms resulting from the data analysis are well within the ensemble spread of the simulations.

One implication of this result is that there is no simple way to adjust the modes estimated with values in the vicinity of the Nyquist lag when the time series is short enough to make those modes highly uncertain. What we can do is look at the time scales associated with the eigenvalues. Figure 8 shows the decay time and period of the most rapidly varying mode as estimated by LIM from the COADS data as function of $\tau_{o}$. We note that these

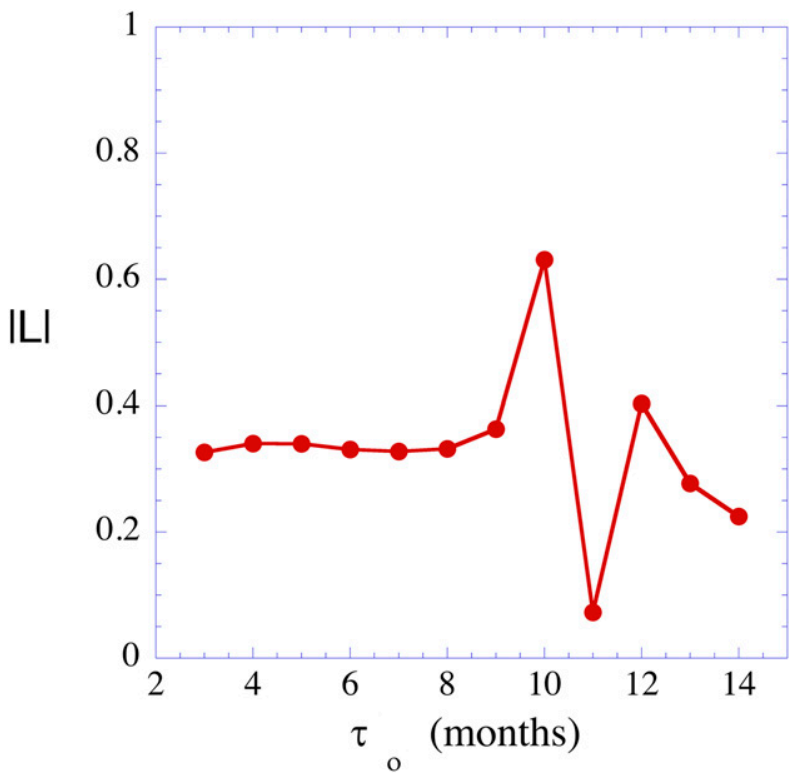

FIG. 6. Euclidean norm of an operator representing a reduced model of El Niño.

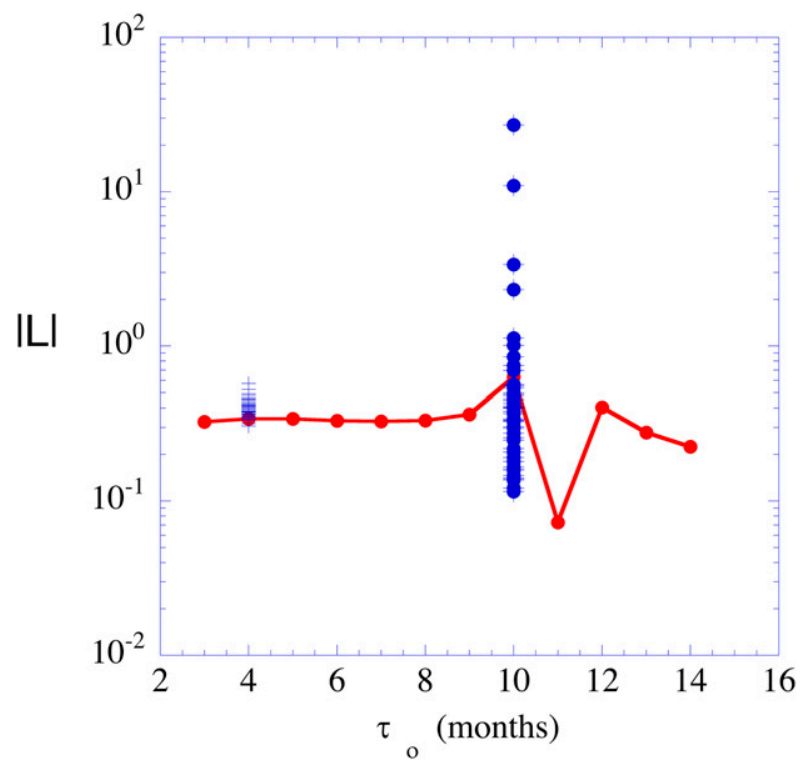

FIG. 7. As in Fig. 6, but on a log plot showing Euclidean norms from a numerically generated ensemble of 50 Ornstein-Uhlenbeck processes evaluated using $\tau_{o}=4$ months (crosses) and $\tau_{o}=10$ months (filled symbols).

curves are flattest when $\tau_{o}$ takes values of 6-8 months, in agreement with Penland and Sardeshmukh's (1995b) result that the eigenvalues are most accurately estimated when the decay time is approximately equal to $\tau_{o}$. We also see that the decay time curve deviates from a flat line much less than the curve of estimated periods, particularly after the Nyquist lag, and that the adjustment to the imaginary part of the eigenvalue suggested above ameliorates the sharp variation of the estimated period with $\tau_{o}$ after $\tau_{o}=11$ months. As for as the modes themselves, it may not be reasonable to estimate them accurately if two thirds of them have decay times less than $\tau_{o}$ although (not shown) they are indeed visually similar to the corresponding complex conjugates of modes estimated for $\tau_{o}$ less than 9 months.

\section{Conclusions}

LIM has been shown to be a useful diagnostic and predictive tool in geophysics specialties as diverse as megadroughts (e.g., Ault et al. 2018, summarized in Physics Today 2018), anthropogenic forcing of global change (e.g., Frankignoul et al. 2017), planetary turbulence (e.g., DelSole and Farrell 1996), monsoon precipitation (e.g., Priya et al. 2015), ocean-atmosphere coupling (e.g., Smirnov et al. 2014), El Niño (e.g., Penland and Sardeshmukh 1995a), and midlatitude low-frequency variability (e.g., Penland and Ghil 1993). An integral part of LIM concerns validation of its underlying assumption 


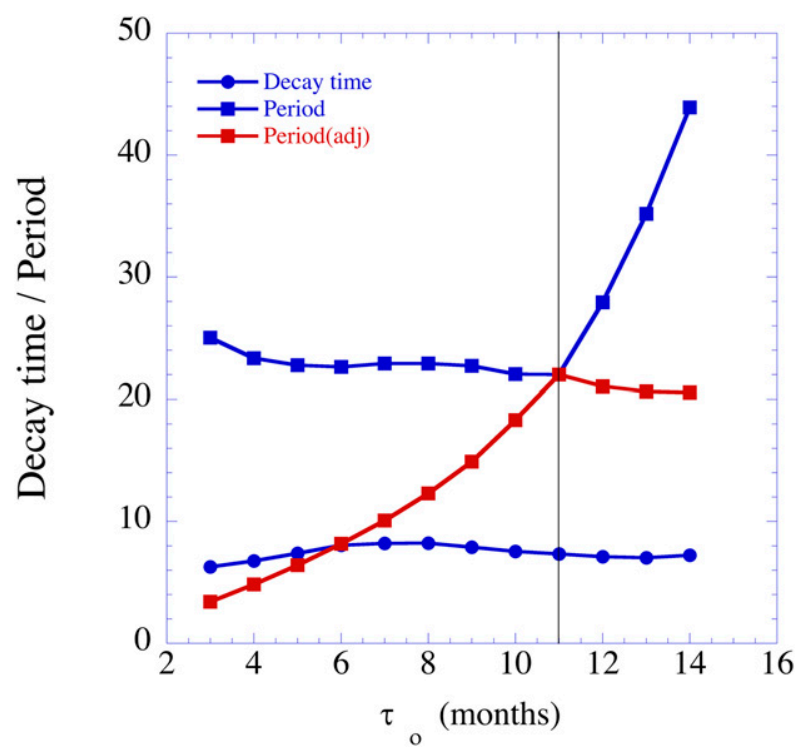

FIG. 8. Raw estimation (blue) of decay times (circles) and periods (squares) in months from COADS data using LIM as a function of $\tau_{o}$. Also shown are the periods resulting from subtracting the estimated angular frequency from $2 \pi$ (red squares). The Nyquist lag at 11 months is indicated by the vertical line.

of linearity. Since the definition of an exponential function is the unique solution of a linear equation, establishment that the autocovariance matrix function of a dataset follows an exponential law is strong indication of linearity. The test for this exponential law is called the "tau test," and, although this test may generally be trusted when it passes, this test has been shown to fail sometimes when it ought to pass.

There are several reasons why failure can occur even when the linearity assumption is true, the most pernicious of which may be the Nyquist issue. Unfortunately, in spite of repeated warnings in the literature, the Nyquist issue in LIM is often underappreciated to the extent that some practitioners of the method have lately expressed some surprise when apprised of it. This article discusses the issue in detail and presents a possible solution when the time series is long enough to apply it; that is, when the time series and the dynamical decay times associated with it are long enough to identify the modes uniquely.

As with all analyses, the LIM technique has its drawbacks, and the Nyquist issue is one of them. The Nyquist issue plagues not only LIM, but other techniques such as generalized equilibrium feedback analysis (GEFA; Liu et al. 2012a) and, as is well known, Fourier analysis. It is as tempting to ignore the limitations of a powerful technique when one uses it as it is to emphasize them when the results are unpopular. Dispassionate analysis requires careful consideration of the techniques used, and we hope that awareness of the Nyquist issue in LIM will help researchers to avoid misinterpretation of LIM results.

Acknowledgments. The author is indebted to the reviewers of this manuscript for their accurate and useful comments.

\section{REFERENCES}

Ault, T. R., S. St. George, J. E. Smerdon, S. Coats, J. S. Manking, C. M. Carillo, B. I. Cook, and S. Stevenson, 2018: A robust null hypothesis for the potential causes of megadrought in western North America. J. Climate, 31, 3-24, https://doi.org/10.1175/ JCLI-D-17-0154.1.

Bronson, R., 1970: Matrix Methods: An Introduction. Academic Press, $284 \mathrm{pp}$.

Cayley, A., 1858: A memoir on the theory of matrices. Philos. Trans., 148, 17-37, https://doi.org/10.1098/rstl.1858.0002.

DelSole, T., 1996: Can quasigeostrophic turbulence be modeled stochastically? J. Atmos. Sci., 53, 1617-1633, https://doi.org/ 10.1175/1520-0469(1996)053<1617:CQTBMS>2.0.CO;2.

_ , and B. F. Farrell, 1996: Quasi-linear equilibration of a thermally maintained, stochastically excited jet in a quasigeostrophic model. J. Atmos. Sci., 53, 1781-1797, https://doi.org/ 10.1175/1520-0469(1996)053<1781:TQLEOA > 2.0.CO;2.

Farrell, B. F., and P. J. Ioannou, 1993: Stochastic dynamics of baroclinic waves. J. Atmos. Sci., 50, 4044-4057, https://doi.org/ 10.1175/1520-0469(1993)050<4044:SDOBW >2.0.CO;2.

Frankignoul, C., G. Gastineau, and Y.-O. Kwon, 2017: Estimation of the SST response to anthropogenic and external forcing and its impact on the Atlantic multidecadal oscillation and the Pacific decadal oscillation. J. Climate, 30, 9871-9895, https://doi.org/10.1175/JCLI-D-17-0009.1.

Hamilton, W. R., 1853: Lectures on Quaternions. Hodges and Smith, $736 \mathrm{pp}$.

Hasselmann, K., 1976: Stochastic climate models. Part I. Theory. Tellus, 28, 473-485, https://doi.org/10.3402/tellusa.v28i6.11316.

Liu, Z., N. Wen, and L. Fan, 2012a: Assessing atmospheric response to surface forcing in the observations. Part I: Cross validation of annual response using GEFA, LIM, and FDT. J. Climate, 25, 6796-6816, https://doi.org/10.1175/JCLI-D11-00545.1.

— , L. Fan, S.-I. Shen, and Q. Liu 2012b: Assessing atmospheric response to surface forcing in the observations. Part II: Cross validation of seasonal response using GEFA and LIM. J. Climate, 25, 6817-6834, https://doi.org/10.1175/JCLID-11-00630.1.

Lorenz, E., 1963: Deterministic non-periodic flow. J. Atmos. Sci., 20, 130-141, https://doi.org/10.1175/1520-0469(1963)020<0130: DNF $>2.0 . \mathrm{CO} ; 2$.

Newman, M., 2007: Interannual to decadal predictability of tropical and North Pacific sea surface temperatures. J. Climate, 20, 2333-2356, https://doi.org/10.1175/JCLI4165.1.

Papanicolaou, G. C., and W. Kohler, 1974: Asymptotic theory of mixing stochastic ordinary differential equations. Commun. Pure Appl. Math., 27, 641-668, https://doi.org/10.1002/ cpa.3160270503.

Penland, C., 1989: Random forcing and forecasting using principal oscillation pattern analysis. Mon. Wea. Rev., 117, 2165-2185, https://doi.org/10.1175/1520-0493(1989)117<2165:RFAFUP $>$ 2.0.CO;2. 
1998: The effect of additive noise on the tau-test. Proc. 22nd Climate Diagnostics Workshop, Berkeley, CA, NOAA, 359-362.

— , and M. Ghil, 1993: Forecasting Northern Hemisphere $700 \mathrm{mb}$ geopotential height anomalies using empirical normal modes. Mon. Wea. Rev., 121, 2355-2371, https://doi.org/10.1175/15200493(1993)121<2355:FNHMGH > 2.0.CO;2.

_- , and P. D. Sardeshmukh, 1995a: The optimal growth of tropical sea surface temperature anomalies. J. Climate, $\mathbf{8}$, 1999-2024, https://doi.org/10.1175/1520-0442(1995)008<1999: TOGOTS $>2.0 . \mathrm{CO} ; 2$.

__ and _ 1995b: Error and sensitivity analysis of geophysical systems. J. Climate, 8, 1988-1998, https://doi.org/10.1175/15200442(1995)008<1988:EASAOG >2.0.CO;2.

— , and L. Matrosova, 2001: Expected and actual errors of linear inverse model forecasts. Mon. Wea. Rev., 129, 1740-1745, https://doi.org/10.1175/1520-0493(2001)129<1740:EAAEOL> 2.0.CO;2.

$\longrightarrow$, and — 2006: Studies of El Niño and interdecadal variability in tropical sea surface temperatures using a nonnormal filter. J. Climate, 19, 5796-5815, https://doi.org/10.1175/JCLI3951.1.

Priya, P., M. Mujumdar, T. P. Sabin, P. Terray, and R. Krishnan, 2015: Impacts of Indo-Pacific sea surface temperature anomalies on the summer monsoon circulation and heavy precipitation over northwest India-Pakistan region during 2010. J. Climate, 28, 3714-3730, https://doi.org/10.1175/JCLI-D-14-00595.1.
Rümelin, W., 1982: Numerical treatment of stochastic differential equations. SIAM J. Numer. Anal., 19, 605-613.

Sheshadri, A., R. A. Plumb, E. A. Lindgren, and D. I. V. Domeison, 2018: The vertical structure of annular modes. J. Atmos. Sci., 75, 3507-3519, https://doi.org/10.1175/JAS-D-17-0399.1.

Shin, S.-I., P. D. Sardeshmukh, and K. Pegion, 2010: Realism of local and remote feedbacks on tropical sea surface temperatures in climate models. J. Geophys. Res., 115, D21110, https:// doi.org/10.1029/2010JD013927.

Smirnov, D., M. Newman, and M. A. Alexander, 2014: Investigating the role of ocean-atmosphere coupling in the North Pacific Ocean. J. Climate, 27, 592-606, https://doi.org/10.1175/JCLID-13-00123.1.

Von Storch, H., T. Bruns, I. Fischer-Bruns, and K. Hasselmann, 1988: Principal oscillation pattern analysis of the 30- to 60-day oscillation in general circulation model equatorial troposphere. J. Geophys. Res., 93, 11 022-11 036, https://doi.org/ 10.1029/JD093iD09p11022.

Winkler, C. R., M. Newman, and P. D. Sardeshmukh, 2001: A linear model of wintertime low-frequency variability. Part I: Formulation and forecast skill. J. Climate, 14, 4474-4494, https://doi.org/10.1175/1520-0442(2001)014<4474:ALMOWL> 2.0.CO;2.

Woodruff, S., S. Lubker, K. Wolter, S. Worley, and J. Elms, 1993: Comprehensive Ocean-Atmosphere Data Set (COADS) Release 1a: 1980-92. Earth Syst. Monit., 4, 3-8. 